\title{
A relevância da territorialização como diferencial na formação médica e na atenção primária à saúde
}

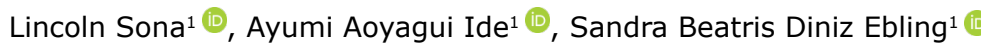

\begin{abstract}
RESUMO
Temas de Ensino em Saúde: a territorialização proporciona uma experiência de imersão de acadêmicos de medicina na realidade da saúde coletiva, oportunizando uma mudança na visão da formação e atuação médica. Além disso, é uma ferramenta para o planejamento em saúde, através do conhecimento do território, da comunidade e da oferta de serviços na atenção primária à saúde. Objetivo: refletir sobre o processo de territorialização como uma estratégia essencial para a ação em saúde no contexto da atenção primária e para a formação médica. Metodologia: Trata-se de um relato de experiência. Após o estudo prévio, realizou-se o processo de territorialização em uma comunidade na região oeste do RS, com posterior discussão dos aprendizados, planejamento e prática da ação em saúde na comunidade. Resultados: foi possível conhecer os moradores e suas condições de vida, quais suas principais necessidades em saúde e criar um senso de responsabilidade nos estudantes quanto à necessidade da participação ativa do médico na comunidade. Conclusão: constata-se a importância da territorialização na oferta de serviços de qualidade à comunidade e que se adéquem às necessidades locais; na criação e manutenção de vínculo entre profissionais de saúde e usuários; e seu impacto na humanização da medicina, na formação crítica e reflexiva de médicos e no estreitamento da relação ensino-serviço-comunidade.
\end{abstract}

Keywords: Educação médica, Medicina, Planejamento em saúde, Saúde pública. 


\section{INTRODUÇÃO}

O Sistema Único de Saúde (SUS) é, de acordo com a Constituição da República Federativa do Brasil, de $1988^{1}$, um sistema público de saúde presente em todo o território nacional que busca garantir acesso universal para toda a população. Apresenta como princípios ${ }^{2}$ : a universalização, que corresponde ao dever do Estado de garantir saúde a todos, sem discriminação; a equidade, que objetiva diminuir desigualdades; e a integralidade, que busca através de integrações com diversos serviços e setores, atender a todas as necessidades dos indivíduos.

O Ministério da Saúde preconiza os princípios organizativos do SUS ${ }^{2}$, são eles: regionalização e hierarquização, que buscam organizar os serviços em ordem crescente de complexidade e de forma articulada; descentralização e comando único, que visam redistribuir poder e responsabilidade entre os três níveis de governo - federal, estadual e municipal - e proporcionar autonomia para cada um deles; e participação popular, que seria o incentivo à população para envolver-se nas questões de saúde.

Nesse contexto, a Atenção Primária à Saúde (APS) merece destaque, por representar o primeiro nível de contato com o sistema público de saúde, sendo a base que promove organização e racionalização de recursos e serviços. Dentre suas funções estão a promoção da saúde, a prevenção e o controle de doenças crônicas, o tratamento e a recuperação de agravos, além do cuidado paliativo. Ela deve funcionar como porta de entrada do sistema, com ações de promoção, manutenção e melhora da saúde, articulando-se com os demais níveis de complexidade e formando uma rede integrada de serviços ${ }^{3}$.

A APS se traduz na Estratégia Saúde da Família (ESF) como um acesso universal e contínuo a serviços de saúde, dentro de um território determinado. A ESF busca aliar a prática do cuidado individual à abordagem populacional na perspectiva da vigilância em saúde, integrar vigilância epidemiológica e sanitária, territorialização/distritalização, atenção clínica e políticas intersetoriais, ações programáticas e reorganização do atendimento à demanda espontânea com acolhimento centrado no usuário, consolidando diretrizes e princípios do SUS como a universalidade, a integralidade e a equidade ${ }^{4}$.
A equipe da ESF realiza ações de promoção, proteção e recuperação da saúde, de acordo com as necessidades da população, as quais são estabelecidas pelo vínculo entre usuários e profissionais em contato com o território ${ }^{5}$, possibilitando, também, estimular a participação popular. Dessa forma, ela representa uma mudança no modelo sanitário brasileiro tradicional, medicamentoso e individual, para um modelo de saúde coletiva vinculado à comunidade ${ }^{6}$, que se propõe a considerar o indivíduo em todos os seus aspectos, sendo eles econômicos, sociais, psicológicos e relacionados diretamente à saúde física.

Através de uma análise mais abrangente do processo saúde-doença, a questão social se evidencia vital, pois é parte intrínseca da vida dos indivíduos. Nesse âmbito, o território representa uma delimitação espacial, um perfil histórico, social, cultural, demográfico, administrativo, tecnológico, político e epidemiológico, o que o caracteriza como um território em permanente construção7. É necessário conhecer e compreender esses aspectos e os principais problemas de saúde da comunidade, para que, assim, as intervenções sociais e de saúde priorizem populações que estão em situação de vulnerabilidade e maior risco ${ }^{8}$.

A territorialização surge como uma ferramenta importante que viabiliza uma análise epidemiológica do território da ESF e de sua população, ou seja, do espaço vivo e dinâmico do ambiente. Uma das diretrizes fundamentais da ESF é a vinculação da população a uma de suas unidades, a partir do estabelecimento de uma base territorial ${ }^{9}$, proporcionando conhecimento do território e de seus moradores. Conhecer as demandas da população possibilita aos profissionais elaborarem políticas de intervenção de saúde, preventivas ou curativas, que melhor atendam às necessidades locais ${ }^{10}$. Consequentemente, favorece a concretização da hierarquização, da regionalização e da participação popular; e contribui para a equidade e a integralidade. Dessa forma, a territorialização atua no planejamento em saúde, colaborando para efetivar princípios do SUS.

No campo do aprender médico, a imersão na APS promove um aprendizado focado na prática e na criação de vínculo entre acadêmicos e comunidades. A territorialização soma ao aprendizado por possibilitar aos discentes uma experiência direta de conhecimento do território, da comunidade, de suas fragilidades e potencialidades. Através desse processo, o estudante de medicina adquire um saber humano da realidade local, o qual, juntamente ao 
saber técnico adquirido na universidade, ajuda-o a expandir sua visão sobre a medicina e a buscar uma formação mais humanizada. Ademais, a inserção de discentes nas ESFs proporciona um estreitamento de relações entre a universidade, o SUS e a comunidade.

Isto posto, segundo Justo ${ }^{11}$, com o planejamento da Educação em Saúde se afastando do modelo biomédico e se adequando à reorientação dos sistemas de saúde, o conhecimento sobre o processo de territorialização se faz ferramenta necessária para uma transição entre tais modelos de modo funcional, principalmente no contexto da APS. A formação acadêmica de profissionais de saúde inseridos nesse âmbito se mostra crítica e libertária, extrapolando domínios técnico-científicos e abrangendo todos os aspectos estruturantes das relações e das práticas nos componentes de interesse e de relevância social ${ }^{12}$. Por conseguinte, evidencia-se o papel da territorialização na inserção de estudantes de medicina no SUS e na comunidade como uma forma de estreitar relações, aprofundar conhecimentos e proporcionar experiências enriquecedoras para a formação médica.

Sendo assim, este artigo tem como objetivo refletir sobre o processo de territorialização como uma ferramenta essencial de planejamento em saúde; para a compreensão das demandas da comunidade; na oferta de serviços específicos para atender às necessidades da população adscrita; na criação, manutenção e estreitamento de vínculo entre os serviços de saúde e os usuários de sua área, no fortalecimento da relação serviço-ensinocomunidade, entre outros fatores positivos advindos do processo; bem como objetiva pautar sobre a formação médica humanizada que, alicerçada na vivência de acadêmicos de medicina na ESF, pode ser enriquecida com a experiência oportunizada pela territorialização.

\section{MATERIAL E MÉTODOS}

Trata-se de um relato de experiência de acadêmicos do terceiro semestre do curso de medicina da Universidade Federal do Pampa (UNIPAMPA), sucedido entre os meses de agosto e dezembro de 2019. Os discentes participaram do planejamento e da realização de um processo de territorialização proposto pelo componente curricular Saúde Coletiva II em uma área ainda não mapeada pela ESF, tratando-se de uma comunidade residente no entorno de um antigo vazadouro da região oeste do Rio Grande do Sul, ampliando o vínculo entre a universidade, representada pelos acadêmicos e docente; o SUS, através dos profissionais de saúde da ESF; e a comunidade, pela participação dos moradores.

Para tanto, dois grupos de alunos de graduação em medicina foram preparados com conceitos e conhecimentos prévios acerca de como é realizada, quais são suas funções e sua importância. Os alunos e a professora responsável foram recebidos pela Agente Comunitária de Saúde (ACS) e a enfermeira da ESF, que explicaram qual região seria alvo da atividade e em quais condições se encontravam seus moradores. Foi também evidenciado o papel fundamental dos ACSs, que atuam na organização da comunidade, na prevenção dos problemas de saúde ou de seu agravamento, promovem a integração da equipe de saúde com a população, entre outras funções. Sendo assim, eles ocupam uma posição fundamental na Política Nacional da Atenção Básica (PNAB), por identificarem os principais problemas que afetam a saúde da comunidade ${ }^{13}$.

Ao longo do semestre, foram realizadas diversas visitas ao território, com o propósito de mapear uma microárea de cobertura da ESF, através de observações participantes dos discentes e visitas domiciliares acompanhadas pela ACS e outros profissionais da ESF. A territorialização foi planejada para reconhecimento do território e das condições de vida da comunidade, sendo norteada por um roteiro de perguntas que continha direcionamentos básicos, entre eles: questionamentos acerca de quem eram os moradores; como viviam; quais dificuldades enfrentavam onde moravam; qual a relação deles com questões de saúde; quais problemas de saúde encontravam no local onde viviam; e quais os aspectos favoráveis da região.

Decorridas as visitas, as informações obtidas foram transferidas para o software Excel para organização e registro dos dados. Através do software Google Earth Pro, localizou-se o território da comunidade alvo com uma visão via satélite. Posteriormente, elaborou-se o mapa do território com as principais informações coletadas durante o processo de territorialização. O mapa produzido foi apresentado em sua versão impressa e disponibilizado em sua versão digital para a equipe da ESF a qual pertence à área em questão. Essa nova ferramenta provida à equipe fornece informações esquadrinhadas da comunidade da microárea e torna possível traçar estratégias e planos de ação para sanar as necessidades específicas dos moradores da região. 
Durante o processo de territorialização, ao final de cada dia de visitação, os alunos se reuniam com a docente responsável e discutiam as vulnerabilidades e as potencialidades encontradas, destacando as principais fragilidades que necessitavam maior atenção e como utilizar as qualidades da comunidade para melhorar as condições de vida de seus moradores. Por meio dessa análise, foi possível planejar ações de saúde cujos objetivos eram diminuir as desigualdades e necessidades da população e valer-se dos pontos positivos encontrados para melhorar as condições de vida da comunidade, não apenas nos aspectos de saúde, mas considerando o ser humano de maneira holística, ou seja, além do panorama saúde-doença, conforme propõe Rabelo ${ }^{14}$.

\section{RESULTADOS E DISCUSSÃO}

Com a intenção de alcançar mudanças no campo da saúde, partindo de um modelo biomédico para um modelo biopsicossocial, a aproximação da universidade aos serviços de saúde, com a introdução de alunos de medicina nas APSs para conhecerem a realidade e as práticas de saúde coletivamente, possui o potencial de construir novos pactos de convivência, efetivando a universalidade dos serviços, da gestão democrática e das práticas integrais de saúde ${ }^{15}$. Para que essa aproximação seja efetiva, as novas Diretrizes Curriculares Nacionais (DCNs) ${ }^{16}$ para o curso de graduação em medicina de 2001, norteadas pelos princípios do SUS, e posteriormente complementadas pelas DCNs de 2014, preconizam o perfil do formando egresso/profissional.

Médico, com formação generalista, humanista, crítica e reflexiva. Capacitado a atuar, pautado em princípios éticos, no processo de saúdedoença em seus diferentes níveis de atenção, com ações de promoção, prevenção, recuperação e reabilitação à saúde, na perspectiva da integralidade da assistência, com senso de responsabilidade social e compromisso com a cidadania, como promotor da saúde integral do ser humano.

Para se atingir a formação médica proposta pelas DCNs, necessitam-se estratégias de ensino que possibilitem ao aluno vivenciar a APS em diversos aspectos, como: no contato direto com usuários do SUS, com as equipes de Saúde da Família e nos ambientes onde esta prática se desenvolve ${ }^{17}$. É preciso que sejam desenvolvidos nos estudantes, nos professores, nos serviços e nas comunidades os valores essenciais à formação da cidadania, possibilitando, assim, uma visão integral do processo saúde-doença e do cuidado com base em seus determinantes físicos, biológicos, psicológicos, socioeconômicos, ambientais, culturais e políticos ${ }^{18}$.

O ensino de Saúde Coletiva atua como um dos meios para se atingir essa formação, sendo o processo de territorialização integrante da proposta do componente curricular Saúde Coletiva II que, por meio da epidemiologia social, aliada às ciências sociais em saúde, visa dar prioridade ao estudo da determinação social e das desigualdades em saúde ${ }^{19}$. Essa disciplina acadêmica tem como objetivo geral compreender a organização e o processo de trabalho na Atenção Básica e reconhecer aspectos biológicos, psicológicos, sociais e ambientais relacionados à saúde dos indivíduos, famílias e comunidades no contexto dos territórios. Quanto aos objetivos específicos, destacam-se: realizar territorialização e construção de mapa vivo identificando potencialidades, riscos e vulnerabilidades presentes no território; estabelecer vínculo com famílias e comunidade; demonstrar empatia e disponibilidade para a escuta, aprimorar habilidades comunicativas e para o trabalho em equipe por intermédio de colaboração e respeito à diversidade; e compreender a importância de conhecer a comunidade para prover serviços específicos de qualidade.

Atentando à importância dos ACSs na criação de uma comunicação efetiva entre profissionais de saúde e a comunidade, no estabelecimento do perfil epidemiológico do território e na manutenção da saúde da população local, cabe ressaltar que, por medida do Governo Federal, a quantidade de ACSs no município foi drasticamente reduzida, o que dificultou a continuidade dos trabalhos de territorialização das microáreas, dentre diversas outras atribuições também prejudicadas. Na ESF em pauta, havia apenas uma ACS, cuja atuação serviu como facilitadora na inserção dos alunos na comunidade e na criação de vínculo entre os profissionais de saúde e os moradores. Nessas condições, a participação dos acadêmicos do curso de medicina foi determinante no processo de territorialização, cuja capacidade de oferecer subsídio para o planejamento de trabalho da ESF nos determinantes e condicionantes do processo saúde-doença ${ }^{20}$ permite atender às necessidades 
da comunidade de forma mais objetiva e completa. Notadamente, uma relação substancial com a comunidade fortifica a confiança nos profissionais de saúde e na saúde pública ${ }^{11}$.

A análise das informações adquiridas por intermédio da territorialização possibilita conhecer as características demográficas; socioeconômicas; as condições de moradia e saneamento básico; a caracterização dos aspectos culturais e religiosos da comunidade; o grau de organização de grupos comunitários; o perfil epidemiológico da população com as principais doenças e agravos que acometem a comunidade, grupos de risco e principais causas de óbitos; as condições de serviços de saúde existentes; e condições do meio ambiente como pavimentação das ruas, vias de acesso, meios de transporte, espaços de lazer, entre outros ${ }^{9}$.

À vista disso, mediante a construção do mapa, foi possível adquirir uma visão esquemática da conjuntura socioeconômica, sanitária e de saúde da região. Ele foi composto pelas casas numeradas, procedimento realizado durante a territorialização, pois as moradias não possuíam numeração, e em cada uma delas as principais informações daquele núcleo familiar. Assim, o mapa se torna um recurso de apoio à qualificação e gestão da informação na atenção primária, pois possibilita monitorar e avaliar indicadores e informações em saúde ${ }^{21}$. Além disso, o mapeamento pode ser utilizado como instrumento didático e gerador de debate com a população sobre suas condições socioeconômicas e territoriais. Nessa perspectiva, ele deve ser produzido valorizando aspectos como determinantes e condicionantes ambientais e sociais e a influência que exercem no desenvolvimento dos agravos de saúde da população ${ }^{22}$. Durante o processo de territorialização abordado neste artigo, buscou-se considerar todos esses aspectos.

A partir da análise das informações da microárea, obtidas por meio da territorialização, a ESF, juntamente com a unidade móvel de saúde do município, discentes de medicina e medicina veterinária e os docentes responsáveis, realizaram uma ação de saúde na comunidade em um sábado pela manhã. Durante esse período, as crianças brincaram com alguns alunos da medicina; às mulheres foi oferecido o exame citopatológico e o exame clínico das mamas; toda a comunidade teve acesso a exames rápidos de infecções sexualmente transmissíveis, como vírus da imunodeficiência humana (HIV), sífilis, hepatite B e hepatite C, vacinação, consulta de enfermagem e consulta médica. Foram distribuídos itens de higiene pessoal e brinquedos, obtidos por doações, preservativos masculinos e femininos e folhetos informativos de saúde disponibilizados pela ESF. Todos esses serviços oferecidos foram acompanhados pelos discentes da medicina através de um sistema de rodízio, para que cada aluno pudesse experienciar as diferentes vivências. Os alunos de medicina veterinária instruíram a população local no que se refere ao cuidado dos animais e à prevenção de saúde quanto a zoonoses, pois a territorialização proporcionou o conhecimento da grande quantidade de animais no território.

Ao final das atividades do componente curricular Saúde Coletiva II, foi proposto um encontro entre os acadêmicos de medicina e a docente para discutir as experiências e os aprendizados adquiridos. Os alunos destacaram a receptividade da equipe da ESF e a disponibilidade para ajudar e ensinar. Salientaram como a visão da medicina pode ser transformada ao se conhecer na prática a saúde coletiva e as necessidades da comunidade com suas especificidades. Percebeuse que não basta o conhecimento técnico-científico, é preciso também atitudes humanizadas, comunicação efetiva, respeito e trabalho em equipe.

Durante as visitas, foi possível notar como o respeito às diferenças, o vocabulário e a linguagem acessíveis e o interesse a todos os aspectos dos indivíduos se faz essencial e atua como diferencial na abordagem e na criação e manutenção de vínculo com os usuários de saúde, isto é, mediante essa inserção no território faz-se possível fortalecer a relação entre ensinoserviço-comunidade. É com cuidado e desconstrução de preconceitos que se efetiva a universalidade à saúde, e é com o reconhecimento das heterogeneidades do povo brasileiro em conjunto ao estudo de modos coerentes de intervenções diante de tal riqueza de diversidade que se busca garantir a equidade ${ }^{12}$.

Dentre as dificuldades, enfatizou-se a necessidade de maior número e frequente capacitação dos ACSs, cuja função na saúde da população possibilita oferecer serviços de saúde eficientes e decisivos para as demandas locais. Ademais, essas experiências proporcionam a consolidação dos conhecimentos teóricos discutidos em sala de aula ${ }^{12}$, demonstram que 0 médico precisa ser um agente ativo no desenvolvimento da comunidade a qual assiste, e possibilitam um entendimento amplo sobre o papel desse profissional na busca de prevenção de agravos e promoção de saúde ${ }^{23}$. 


\section{CONCLUSÕES}

A territorialização é uma importante estratégia para a efetivação das funções do SUS, contribuindo para o planejamento em saúde e é essencial e premissa básica para o funcionamento da ESF. Faz-se primordial para o reconhecimento do perfil epidemiológico de um território, de suas características demográficas e socioeconômicas, de infraestrutura e lazer, das doenças e agravos predominantes na área e na oferta de serviços que atendam às necessidades específicas da comunidade, permitindo a construção de vínculo entre os profissionais de saúde junto aos usuários com compreensão ampliada do processo saúde-doença.

A experiência oportunizada pelo processo amplifica o impacto da atuação dos acadêmicos de medicina na APS, proporcionando um aprofundamento na vivência com a comunidade; na criação de vínculo com os usuários da saúde pública; no trabalho em equipe com profissionais de saúde de diferentes áreas na ESF, como enfermeiros, técnicos em enfermagem, médicos e odontólogos; e na percepção da importância de um cuidado multiprofissional interdisciplinar. Essa imersão na saúde pública, através de processos como a territorialização e de componentes curriculares como a Saúde Coletiva, expande o saber médico técnicocientífico para um saber humano, que considera o usuário de saúde em todos os seus aspectos e trabalha para que ele se envolva e seja atuante em sua própria saúde e na saúde de sua comunidade.

As vivências no território evidenciam as condições de vida da população local, suas vulnerabilidades e sua dependência dos serviços públicos de saúde, assim como demonstram os potenciais da comunidade e como escutar suas necessidades e ideias pode ser decisivo na adequação da oferta de serviços efetivos e de qualidade e fortificam a atuação popular na saúde, estreitando o elo entre universidade, comunidade e saúde pública.

À vista disso, a vivência da territorialização favorece a compreensão e a concretização de princípios do SUS, como a regionalização, a hierarquização, a participação popular, a integralidade e a equidade da saúde pública. A atividade ainda proporciona uma formação acadêmica médica mais reflexiva, crítica e humanizada; revela a responsabilidade do médico em relação às singularidades e particularidades da população, para posterior articulação dessas questões nas atividades de prevenção e promoção de saúde; e solidifica o processo de integração serviçoensino-comunidade.

\section{REFERÊNCIAS BIBLIOGRÁFICAS}

1. Brasil. Constituição da República Federativa do Brasil de 1988. Seção II, Da Saúde; Art. 196. [Capturado 16 abr 2020]. Disponível em: http://www. planalto.gov.br/ ccivil_03/constituicao/constituicao.htm.

2. Brasil. Ministério da Saúde. Princípios do SUS. Portal do ministério da saúde. [Acesso em 2020 fev 19]. Disponível em: https://www.saude.gov.br/sistemaunico-de-saude/principios-do-sus.

3. Starfield B. Atenção primária: equilíbrio entre necessidades de saúde, serviços e tecnologia, Brasília: UNESCO, Ministério da Saúde, 726p. 2002.

4. Giovanella L, Franco CM, Almeida PF. Política Nacional de Atenção Básica: para onde vamos?. Ciênc. saúde coletiva. Abr 2020 [acesso em 2020 jul 6]; v. 25, n. 4, p. 1475-82. Disponível em: https://www.scielo.br/pdf/ csc/v25n4/1413-8123-csc-25-04-1475.pdf.

5. Oliveira MAC, Pereira IC. Atributos essenciais da Atenção Primária e a Estratégia Saúde da Família. Rev. bras. enferm.. Set 2013 [acesso em $2020 \mathrm{fev} \mathrm{19];} \mathrm{v.}$ 66, n. spe, p. 158-64. Disponível em: http://www. scielo.br/scielo.php?script=sci_arttext\&pid=S0034$71672013000700020 \&$ lng =en\&nrm=iso.

6. Costa GD, Cotta RMM, Ferreira MLSM, Reis JR, Franceschini SCC. Saúde da família: desafios no processo de reorientação do modelo assistencial. Rev. bras. enferm.. Fev 2009 [acesso em 2020 jul 28]; v. 62 , n. 1, p. 113-8. Disponível em: http://www. scielo.br/scielo.php?script $=$ sci_arttext\&pid =S0034$71672009000100017 \&$ lng $=$ en\&nrm $=$ iso.

7. Gondim GMM, Monken M, Rojas LI, Barcellos C, Peiter P, Navarro M, Gracie R. O território da saúde: a organização do sistema de saúde e a territorialização. Território, ambiente e saúde, Editora Fiocruz. 2008 [acesso em 2020 jul 9]; p. 237-55. Disponível em: http://www.escoladesaude.pr.gov.br/arquivos/File/ TEXTOS_CURSO_VIGILANCIA/20.pdf

8. Furlan PG. Veredas no território: análise da prática de Agentes Comunitários de Saúde. Campinas, 2008. Dissertação (Mestrado em Saúde Coletiva). Faculdade de Ciências Médicas, UNICAMP.

9. Aquino R. Conhecendo o território. Manual para treinamento introdutório das equipes de saúde da família., Bahia. Polo de Capacitação, Formação e Educação Permanente de Pessoal para a Saúde da Família. 2001 [acesso em 2020 jul 8]; p. 183, 2001. Série Cadernos Técnicos, 02. Texto de Apoio 3. Disponível em: https://edisciplinas. usp.br/pluginfile.php/4608315/mod_resource/content/1/ Conhecendo\%200\%20Territ\%C3\%B3rio.pdf.

10. Arantes LJ, Shimizu HE, Merchán-Hemann E. Contribuições e desafios da Estratégia Saúde da Família na Atenção Primária à Saúde no Brasil: revisão da literatura. Ciência \& Saúde Coletiva. 2016 [acesso em 2020 fev 18]; v. 21, n. 5, p. 1499-510. Disponível em: http://www.scielo.br/scielo. php?script=sci_arttext\&pid=S1413-81232016000501499. 
11. Araújo GB, Alves Filho FWP, Santos RS, Lira RCM. Territorialização em saúde como instrumento de formação para estudantes de medicina: relato de experiência. SANARE. Jan./jul. 2017 [acesso em 2020 fev 20]; v.16, n.01, p. 124-9. Disponível em: https:// sanare.emnuvens.com.br/sanare/article/view/1103.

12. Justo LG, Severo AKS, Félix-Silva AV, Soares LS, SilvaJúnior FL, Pedrosa JIS. A territorialização na Atenção Básica: um relato de experiência na formação médica. Interface Botucatu. 2017 [acesso em 2020 fev 19]; v. 21, supl. 1, p. 1345-54. Disponível em: http://www. scielo.br/scielo.php?script=sci_arttext\&pid $=$ S1414$32832017000501345 \&$ lng $=$ en\&nrm $=$ iso

13. Nunes MO, Trad LB, Almeida BA, Homem CR, Melo MCIV. O agente comunitário de saúde: construção da identidade desse personagem híbrido e polifônico. Cad. Saúde Pública. 2002 [acesso em 2020 jun 15]; v. 18, n. 6, p. 1639-46. Disponível em: https://repositorio.ufba. br/ri/bitstream/ri/3678/1/13260.pdf.

14. Rabello E, Bandeira LLB, Anjos ILPB, Santos CT, Macêdo TLS, Silva CPO, et al. Experiência com a prática de humanização na relação médico-paciente em alunos do primeiro período do curso de Medicina na Universidade de Vassouras. Rev. Fluminense de Extensão Universitária. 2019 [acesso em 2020 mar 1]. Disponível em: http://editora.universidadedevassouras. edu.br/index.php/RFEU/article/view/1793.

15. Anjos RMP, Gianini RJ, Minari FC, Luca AHS, Rodrigues MP. "Vivendo o SUS": uma experiência prática no cenário da atenção básica. Rev. bras. educ. med.. Mar 2010 [acesso em 2020 jul 7]; v. 34, n. 1, p. 172-83. Disponível em: https:// www.scielo.br/scielo.php?script=sci_arttext\&pid=S010055022010000100021\&lng=pt\&tlng=pt.

16. Brasil. Ministério da Educação, Conselho Nacional de Educação, Câmara da Educação Superior. Parecer no 1.133 , de 7 de agosto de 2001. Diretrizes Curriculares Nacionais dos cursos de graduação em Enfermagem, Medicina e Nutrição. 2001 [acesso em 2020 jul 6]. Disponível em: http://portal.mec.gov.br/dmdocuments/ ces1133.pdf.
17. Souza CFT, Oliveira DLL, Monteiro GS, Barboza HMM, Ricardo GP, Lacerda Neto MC, et al.. A atenção primária na formação médica: a experiência de uma turma de medicina. Rev. bras. educ. med.. Set 2013 [acesso em 2020 jul 7]; v. 37, n. 3, p. 448-54. Disponível em: https://www.scielo.br/pdf/rbem/v37n3/18.pdf.

18. Gomes AP, Costa JRB, Junqueira TS, Arcuri MB, SiqueiraBatista R. Atenção primária à saúde e formação médica: entre episteme e práxis. Rev. bras. educ. med.. Dez 2012 [acesso em 2020 jul 7]; v. 36, n. 4, p. 541-9. Disponível em: https://www.scielo.br/scielo.php?script=sci_arttext\& pid $=$ S0100-55022012000600014.

19. Souza LE. Presidente da Abrasco fala sobre $11^{\circ}$ Abrascão. Portal da ABRASCO, Associação Brasileira de Pós-Graduação em Saúde Coletiva. Jul. 2015 [acesso em 2020 fev 27]. Disponível em: https://www.abrasco.org. $\mathrm{br} / \mathrm{site} /$ outras-noticias/opiniao/presidente-da-abrascofala-sobre-11o-abrascao/12157.

20. Tetemann EC, Trugilho SM, Sogame LCM. Universalidade e Territorialização no SUS: contradições e tensões inerentes. Textos \& Contextos. 2016 [acesso em 2020 fev 21]; v. 15, n. 2, p. 356-69. Disponível em: http://revistaseletronicas. pucr/.

21. Pinto LF, Rocha CMF. Inovações na Atenção Primária em Saúde: o uso de ferramentas de tecnologia de comunicação e informação para apoio à gestão local. Ciência \& Saúde Coletiva. 2016 [acesso em 2020 mar 1]; v. 21, n. 5, p. 1433-48. Disponível em: http://www.scielo.br/scielo. php?pid =S1413-81232013000800009\&script $=$ sci_ abstract\&tlng $=$ pt.

22. Goldstein RA, Barcellos C. Geoprocessamento e Participação Social: ferramentas para a vigilância ambiental em saúde. In: Território, Ambiente e Saúde, Editora Fiocruz. 2008.

23. Rodrigues JO. Territorialização em saúde: Um relato de experiência. CONBRACIS, II Congresso Brasileiro de Ciência e Saúde, Campina Grande. 14 a 16 jun 2017 [acesso em 2020 fev 20]. Disponível em: http://www.editorarealize. com.br/revistas/conbracis/trabalhos/TRABALHO_EV071_ MD4_SA7_ID1474_14052017222453.pdf. 
Territorialização: formação médica e atenção primária à saúde

Autor Correspondente:

Lincoln Sona

lincolnsona@gmail.com

Editor:

Prof. Dr. Felipe Villela Gomes

Recebido: $21 / 08 / 2020$

Aprovado: $15 / 10 / 2020$ 